Makuch Marcelina, Makuch Marcin, Krzewicka-Romaniuk Ewa, Milanowski Janusz. Obstructive sleep apnea - risk factors, diagnosis and management. Journal of Education, Health and Sport. 2020;10(9):121-125. eISSN 2391-8306. DOI http://dx.doi.org/10.12775/JEHS.2020.10.09.014

https://apcz.umk.pl/czasopisma/index.php/JEHS/article/view/JEHS.2020.10.09.014

https://zenodo.org/record/4016750

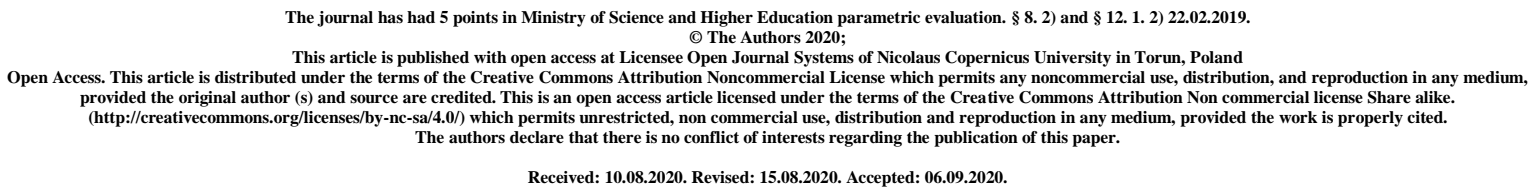

\title{
Obstructive sleep apnea - risk factors, diagnosis and management
}

\author{
Marcelina Makuch, Marcin Makuch, Ewa Krzewicka-Romaniuk, Janusz Milanowski
}

Marcelina Makuch

ORCID iD https://orcid.org/0000-0002-6850-7006

Affiliation Medical University of Lublin. Chair and Department of Pneumology, Oncology and Allergology.

Country Poland

Bio Statement -

Principal contact for editorial correspondence.

Marcin Makuch

ORCID iD https://orcid.org/0000-0001-7139-9862

Affiliation Medical University of Lublin. Chair and Department of Internal Diseases.

Country Poland

Bio Statement -

Ewa Krzewicka-Romaniuk

ORCID iD https://orcid.org/0000-0002-7205-365X

Affiliation Medical University of Lublin. Chair and Department of Pathophysiology.

Country Poland

Bio Statement -

Janusz Milanowski

ORCID iD https://orcid.org/0000-0002-8616-596X

Affiliation Medical University of Lublin. Chair and Department of Pneumology, Oncology and Allergology.

Country Poland

Bio Statement - 


\section{Abstract \\ OBSTRUCTIVE SLEEP APNEA}

Obstructive sleep apnea (OSA) is a sleep-related breathing disorder that is characterised by obstructive apneas, hypopneas and respiratory effort-related arousals caused by repetitive collapse of the upper airway during sleep. Most pauses in breathing last between 10 and 30 seconds, but some may persist longer [1].

Key words: obstructive sleep apnea; snoring; polisomnography; CPAP

\section{RISK FACTORS}

The major risk factors for OSA include:

1) older age

2) obesity

3) male gender

4) craniofacial abnormalities

5) upper airway abnormalities

6) nasal congestion

7) smoking

8) alcohol

9) family history of snoring

Anatomical changes that contribute to oropharyngeal space reduction are among the most important risk factors of OSA. Patients with increased neck circumference and craniofacial alterations - such as increased tongue base, amygdala and uvula - or maxillomandibular deficiencies are at greater risk for apnea, because there is a reduction in the lumen of the upper airways. Sleeping in the supine position also facilitates the occurrence of apneas due to the posterior repositioning of the tongue by gravitational effect.

What is more, OSA often coexists with the following comorbidities: obesity hypoventilation syndrome, congestive heart failure, hypertension, end-stage renal disease, type 2 diabetes mellitus, chronic obstructive pulmonary disease, stroke, pregnancy, hypothyroidism, acromegaly, polycystic ovary syndrome, Parkinson's disease, gastroesophageal reflux disease. Some medications may exacerbate OSA, e.g. benzodiazepines, narcotics, alcohol

Patients with OSA are at increased risk for: driving and motor vehicle crashes, cardiovascular and cerebrovascular morbidity, neuropsychiatric dysfunction, right heart failure, metabolic syndrome $[2,3]$. 


\section{SYMPTOMS}

Most patients with OSA complain with:

1) daytime sleepiness

2) loud snoring, choking or gasping during sleep

3) morning headaches

4) sleep maintenance insomnia

5) nocturia

During physical examination the following findings draw attention:

1) obesity

2) anatomical changes in oropharyngeal airway: retrognathia, micrognathia, lateral peritonsillar narrowing, macroglossia, tonsillar hypertrophy, an elongated or enlarged uvula, a high arched or narrow palate, nasal septal deviation, and nasal polyps

3) large neck circumference $[4,5]$.

Screening questionnaires are evaluation tools to search for patients at risk of OSA. However, their validity in practice still remains weak, because they may detect OSA in highly symptomatic patients. The most common questionnaires used by physicians include the following:

1) the Epworth Sleepiness Scale (ESS) - a simple questionnaire measuring the probability of falling asleep in a variety of situations. The conceptual basis of the ESS involves a fourprocess model of sleep and wakefulness [6].

2) the STOP-Bang - an eight-item survey that incorporates information on snoring, tiredness, observed apneas (obstruction), blood pressure, body mass index, age, neck circumference and gender. A score of three or higher identifies people with OSA [7].

3 ) the sleep apnea clinical score (SACS) - The SACS is a four-item questionnaire that incorporates information on neck circumference, hypertension, habitual snoring, and nocturnal gasping or choking to generate a score ranging from 0 to 100 . It reliably predicts OSA for patients in family medicine practice: scores greater than 15 result in a probability of OSA of 25 to 50 percent [8].

4) the Berlin questionnaire - The Berlin questionnaire consists of 10 items relating to snoring, nonrestorative sleep, sleepiness while driving, apneas during sleep, hypertension, and BMI. The results stratify patients as having a high or low risk for OSA [9].

5) the NoSAS score - The NoSAS score assigns points based upon five parameters (neck circumference, body mass index, snoring, age, and gender). In a derivation and validation analysis, a score $\geq 8$ identified individuals at risk of clinically significant sleep-disordered breathing [10]. 


\section{DIAGNOSIS}

Polisomnography (PSG) remains the gold-standard diagnostic test for OSA.

In patients who undergo PSG, the diagnosis of OSA is confirmed if either of the two criteria below are met:

1) There are 15 or more apneas, hypopneas, or RERAs per hour of sleep (ie, an AHI or respiratory disturbance index $[\mathrm{RDI}] \geq 15$ events per hour) in an asymptomatic patient.

2) There are five or more obstructive apneas, obstructive hypopneas, or RERAs per hour of sleep (ie, an AHI or RDI $\geq 5$ events per hour) in a patient with the following: sleepiness, nonrestorative sleep, fatigue, or insomnia symptoms; waking up with breath holding, gasping, or choking; habitual snoring, breathing interruptions, or both noted by a bed partner or other observer; and/or hypertension, mood disorder, cognitive dysfunction, coronary artery disease, stroke, congestive heart failure, atrial fibrillation, or type 2 diabetes mellitus [11].

Polysomnographic subtypes, distinguished by respiratory event association with hypoxemia, arousals, or both, exhibit varying risks of cardiovascular disease and potential response to therapy [12].

\section{TREATMENT}

Behavior modification, which is indicated for most patients with OSA includes:

1) losing weight (if overweight or obese)

2) exercising

3) changing the sleep position (if OSA is positional)

4) abstaining from alcohol

5) avoiding certain medications eg. benzodiazepines, barbiturates, antiepileptic drugs, sedating antidepressants, antihistamines, opiates.

Positive airway pressure therapy is recommended as initial therapy for adults with OSA. The mechanism of continuous positive airway pressure (CPAP) involves maintenance of a positive pharyngeal transmural pressure so that the intraluminal pressure exceeds the surrounding pressure. CPAP also stabilizes the upper airway through increased endexpiratory lung volume. As a result, respiratory events due to upper airway collapse (eg, apneas, hypopneas) are prevented. [13, 14, 15].

OSA is a heterogeneous disorder. There are 3 basic subtypes replicated in multiple studies:

1) a group where insomnia is the main symptom;

2) an asymptomatic group;

3) a group with marked excessive sleepiness.

The symptomatic benefit from treatment with nasal CPAP varies between these 3 subtypes. Data from the Sleep Heart Health Study reveal that the increased risk of cardiovascular disease from OSA occurs only in the excessively sleepy group [16].

Other treatments include dental devices and surgery. According to Chapman et al. pharmacologic therapy (with agents such as modafinil or armodafinil) may be beneficial as adjunctive therapy for excessive daytime sleepiness that persists despite documentation of adequate and successful conventional therapy (eg, positive airway pressure, oral appliances) [17]. 
Liu et al. discovered that acetazolamide improves sleep apnea at high altitude by decreasing AHI and percentage of periodic breathing time and increasing nocturnal oxygenation. Acetazolamide is more beneficial in healthy participants than in OSA patients, and a $250 \mathrm{mg}$ daily dose may be as effective as higher daily doses for healthy trekkers [18].

Upper airway stimulation (UAS) has been shown to reduce severity of OSA. In a large multicentre international registry, UAS is an effective treatment option with high patient satisfaction and low adverse events. Increasing age and reduced BMI are predictors of treatment response [19].

\section{REFERENCES}

1. https://aasm.org/resources/factsheets/sleepapnea.pdf

2. Jennum P, Riha RL. Epidemiology of sleep apnoea/hypopnoea syndrome and sleep-disordered breathing. Eur Respir J 2009; 33:907.

3. Faber J, Faber C, Faber A. Obstructive sleep apnea in adults. Dental Press J Orthod. 2019;24(3):99-109

4. Zieliński J, Pływaczewski R, Bednarek M. Zaburzenia oddychania w czasie snu. PZWL, Warszawa 2005: 57-65.

5. Olszewska E. Chrapanie i bezdechy. Diagnostyka i leczenie. Medipage. Warszawa 2019: 35-50.

6. Johns MW. Daytime sleepiness, snoring, and obstructive sleep apnea. The Epworth Sleepiness Scale. Chest 1993; 103:30.

7. Chiu HY, Chen PY, Chuang LP, et al. Diagnostic accuracy of the Berlin questionnaire, STOP-BANG, STOP, and Epworth sleepiness scale in detecting obstructive sleep apnea: A bivariate meta-analysis. Sleep Med Rev 2017; 36:57.

8. Grover M, Mookadam M, Chang YH, Parish J. Validating the Diagnostic Accuracy of the Sleep Apnea Clinical Score for Use in Primary Care Populations. Mayo Clin Proc 2016; 91:469.

9. Netzer NC, Stoohs RA, Netzer CM, et al. Using the Berlin Questionnaire to identify patients at risk for the sleep apnea syndrome. Ann Intern Med 1999; 131:485.

10. Marti-Soler H, Hirotsu C, Marques-Vidal P, et al. The NoSAS score for screening of sleep-disordered breathing: a derivation and validation study. Lancet Respir Med 2016; 4:742.

11. American Academy of Sleep Medicine. International Classification of Sleep Disorders, 3rd ed, American Academy of Sleep Medicine, Darien, IL 2014.

12. Zinchuk A, Yaggi HK. Phenotypic Subtypes of OSA: A Challenge and Opportunity for Precision Medicine. Chest. 2020;157(2):403-420.

13. Patil SP, Ayappa IA, Caples SM, Kimoff RJ, Patel SR, Harrod CG. Treatment of adult obstructive sleep apnea with positive airway pressure: an American Academy of Sleep Medicine clinical practice guideline. $J$ Clin Sleep Med. 2019;15(2):335-343.

14. Tażbirek M, Pierzchała W. Zaburzenia oddychania podczas snu w praktyce. PZWL. Warszawa 2019: 40-55.

15. Jordan AS, McSharry DG, Malhotra A. Adult obstructive sleep apnoea. Lancet 2014; 383:736.

16. Pack AI. Further Development of P4 Approach to Obstructive Sleep Apnea. Sleep Med Clin. 2019;14(3):379-389.

17. Chapman JL, Vakulin A, Hedner J, et al. Modafinil/armodafinil in obstructive sleep apnoea: a systematic review and meta-analysis. Eur Respir J 2016; 47:1420.

18. A Liu HM, Chiang IJ, Kuo KN, Liou CM, Chen C. The effect of acetazolamide on sleep apnea at high altitude: a systematic review and meta-analysis. Ther Adv Respir Dis. 2017;11(1):20-29.

19. Heiser C, Steffen A, Boon M, et al. Post-approval upper airway stimulation predictors of treatment effectiveness in the ADHERE registry. Eur Respir J. 2019;53(1):1801405. 